\title{
A specific insertion of a solo-LTR characterizes the Y-chromosome of Bryonia dioica (Cucurbitaceae)
}

\author{
Ryan K Oyama*, Martina V Silber and Susanne S Renner
}

\begin{abstract}
Background: Relatively few species of flowering plants are dioecious and even fewer are known to have sex chromosomes. Current theory posits that homomorphic sex chromosomes, such as found in Bryonia dioica (Cucurbitaceae), offer insight into the early stages in the evolution of sex chromosomes from autosomes. Little is known about these early steps, but an accumulation of transposable element sequences has been observed on the $Y$ chromosomes of some species with heteromorphic sex chromosomes. Recombination, by which transposable elements are removed, is suppressed on at least part of the emerging Y-chromosome, and this may explain the correlation between the emergence of sex chromosomes and transposable element enrichment.

Findings: We sequenced 2321 bp of the Y-chromosome in Bryonia dioica that flank a male-linked marker, BdY1, reported previously. Within this region, which should be suppressed for recombination, we observed a solo-LTR nested in a Copia-like transposable element. We also found other, presumably paralogous, solo-LTRs in a consensus sequence of the underlying Copia-like transposable element.

Conclusions: Given that solo-LTRs arise via recombination events, it is noteworthy that we find one in a genomic region where recombination should be suppressed. Although the solo-LTR could have arisen before recombination was suppressed, creating the male-linked marker $B d Y 1$, our previous study on $B$. dioica suggested that $B d Y 1$ may not lie in the recombination-suppressed region of the Y-chromosome in all populations. Presence of a solo-LTR near $B d Y 1$ therefore fits with the observed correlation between retrotransposon accumulation and the suppression of recombination early in the evolution of sex chromosomes. These findings further suggest that the homomorphic sex chromosomes of $B$. dioica, the first organism for which genetic $X Y$ sex-determination was inferred, are evolutionarily young and offer reference information for comparative studies of other plant sex chromosomes.
\end{abstract}

\section{Background}

The origin and evolution of sex chromosomes from autosomes have long interested biologists. Dioecy, a precondition for sex chromosomes where the male and female functions are separated onto different individuals, is common in animals but relatively rare among flowering plants, occurring in only about $6 \%$ of species [1]. Sex chromosomes in flowering plants are even less common, with only a handful of species known to have them $[2,3]$. Molecular clock dating and the observation that some of these species (e.g., papaya) still have homomorphic gonosomes, suggest that plant sex chromosomes are evolutionarily young $[4,5]$.

* Correspondence: royama@lrz.uni-muenchen.de

1 Systematic Botany \& Mycology, Ludwig-Maximilians-Universität (LMU Munich), 80638 Munich, Germany

Full list of author information is available at the end of the article
Most of what we know about the evolution of sex chromosomes in flowering plants is derived from research on a few model systems. Molecular level investigations in Rumex, Silene and Carica have yielded a wealth of data [6]. In Fragaria, there is evidence of at least some recombination between the sex-determining loci, a hallmark of incipient sex chromosomes, offering support for models of the gradual evolution of sex chromosomes $[4,7,8]$. Nevertheless, the early steps in the evolution of sex chromosomes from autosomes remain unclear as direct evidence is rare [9]. An empirical correlation observed is the accumulation of transposable elements and other repetitive sequences on the Y- (or W-) chromosome [10].

Transposable elements are ubiquitous components of all genomes. In angiosperms, they make up a particularly large component of genomes and are mostly long-terminal repeat (LTR) retrotransposons in the Copia or Gypsy 
superfamilies [11]. Like all retrotransposons, LTR-retrotransposons move intracellularly by a replicative mechanism similar to that of retroviruses and transpose through reverse transcription of an RNA intermediate. LTR-retrotransposons in particular tend to transpose into other LTR-retrotransposons [12-14]. Removal of retrotransposon DNA from the plant genome is thought to require recombination [15]. One such mechanism for removal, unequal homologous recombination, involves recombination between the two LTRs of an LTR-retrotransposon resulting in a solo-LTR [16], making the presence of a solo-LTR a signal of recombination. Conversely, one would expect accumulation of retrotranspon sequences, but not solo-LTRs, where recombination is suppressed, such as on the Y-chromosome [17]. The emerging picture appears, however, to be more complex.

In Silene latifolia, Copia-like retrotransposons are not preferentially accumulated on the sex chromosomes, but are enriched on the $\mathrm{Y}$ - compared to the X-chromosome [18-20]. In Rumex acetosa, specific transposable elements accumulate on the two versions of the Y-chromosome $[21,22]$. Non-LTR retroelements have been found in the terminal region of the long arm of the Y-chromosome of Cannabis sativa [23]. In Carica, which has homomorphic sex chromosomes, the male-specific Y-region is differentiated from the corresponding region on the X-chromosome by an accumulation of transposable elements and inverted repeats [24], suggesting that retrotransposon accumulation begins very early in the establishment of sex chromosomes. Here we report findings from Bryonia dioica (Cucurbitaceae), a species with homomorphic sex chromosomes, in which we have found a complex association of two LTR-retrotransposons.

The cucurbit Bryonia dioica was the first organism for which an XY sex-determination system was inferred from the sex ratios obtained in reciprocal crosses between the dioecious B. dioica and the monoecious B. alba $[25,26]$. A phylogeny [27] and biogeographic study [28] of the ten species of Bryonia indicate that dioecy may have reevolved in the lineage leading to B. dioica, implying that the homomorphic sex chromosomes are evolutionarily young (i.e., a few million years old). In our previous paper [29], we found that the male-linked marker $B d Y 1$ may not lie in the zone of recombination-suppression on the Ychromosome in all populations, suggesting a recent origin. A second marker, $B d X 1$, was common to both male and females, but was highly similar in sequence to $B d Y 1$, differing mainly by the presence of a large insertion. In this study we used $B d Y 1$ as an anchor point to perform chromosome walking along the Y-chromosome of $B$. dio$i c a$, which revealed a Copia-like element within which a solo-LTR is inserted. We also recovered the consensus sequence of the underlying Copia-like retrotransposon via genome walking within which we found solo-LTRs inserted at multiple, presumably paralogous locations, one of which created $B d Y 1$ and thus characterizes the Ychromosome.

\section{Results}

We sequenced $415 \mathrm{bp}$ upstream and $1649 \mathrm{bp}$ downstream of the SCAR marker BdY1 described in Oyama et al. [29] on the Y-chromosome of Bryonia dioica. Within this sequence (hereafter CW278-Y), which has been submitted to GenBank [GenBank:HM365927], we found an LTR-retrotransposon. We identified the open reading frame (ORF) and, using specific primers for the coding region, continued sequencing the retrotransposon via genome walking. This consensus sequence of the underlying retrotransposon (hereafter RLC-sequence) had a final consensus length of 5008 bp [GenBank:HM365926].

A BLASTn [30,31] search in the TREP database [32] with CW278-Y found the highest similarity to an element from the Angela family of LTR-retrotransposons in the Copia superfamily [33], while a BLASTn search in GenBank yielded similarity to a Copia-like element from Cucumis (Cucurbitaceae) [GenBank:GQ326556]. BLASTx analyses of the translated amino acid sequence from the RLC-sequence in GenBank also found similarity to elements in the Copia superfamily. Conserved domain search analysis [34] demonstrated that this transposable element encodes a polyprotein with a $\mathrm{CCHC}$ zinc knuckle (gag) domain (position 1890-1934 on the RLCsequence, amino acids 254-268), an integrase core (rve) domain (position 2616-3098 on the RLC-sequence, aa 496-656) and a reverse transcriptase (RVT-2) domain (position 3600-4361 on the RLC-sequence, aa 823-1077). Because we could not identify a protease domain, we assume that this element is inactive. That the domains within the polyprotein ( $p o l)$ gene are arranged in the order rve/RVT also supports assignment to the Copia superfamily.

A putative solo-LTR of 772 bp was identified in CW278-Y beginning at position 584 of the sequence. This was done by aligning the CW278-Y sequence to the Copia-like element from Cucumis found in BLASTn searches (see Figure 1). The solo-LTR is flanked by 5 -bplong direct but incomplete repeats (GTCGG at the 5 'end and GTCG at the 3'end) representing the target-site duplication and contains the $5^{\prime}$-terminal and $3^{\prime}$-terminal sequences 5 ' TG...CA 3'. This insertion event corresponds to the male-linked SCAR marker $B d Y 1$ described in Oyama et al. [29]. Multiple, presumably paralogous insertion sites within the RLC-sequence could be detected by PCR using internal primers specific to the solo-LTR, the 5 -LTR and the coding region. One of these additional events corresponds to the $B d X 1$ sequence described in Oyama et al. [29] and the remainder were named $\alpha, \beta, \gamma$ and $\delta$ (see Figure 2). 


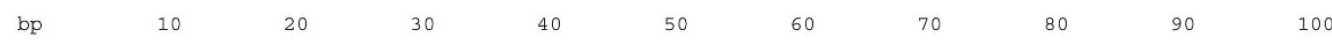

GQ326556 AAACA---TATCTTCCATTTTTC--ATAG---AAATAGGTCGATATCTTGTAAAACT--GGTTACAA-AAGGCTCACCTGGTTCAATCTTGTCAACAAGT

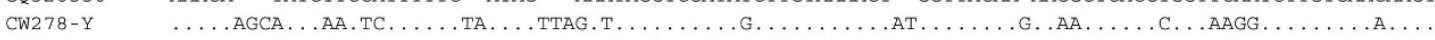
GQ326556 CAGATAAGATGACTAAGGTTGGAGGTTCTTAAGTTGACGGTTTACGGAACACCTCCTACCTGGAGATCGTAATCAGTTCTTGAGCCTAGTTAACCTAGTT CW278-Y GQ326556 TTATGAGCATTCGTGAGAGGTGTGAGGAAATAAAAATGGTTTTTCATCTAGACATCATAAGTTAAAATCCTGTATAATGGTTATACTTGGATGTTTTATC TAGACTTAAGATTTGTTTAAGTTAGCCTAAATATAAATCTTGAAGCTTGGATTACCCTATTTTTTTTCTCTAGAACACTTCAACTAGAGAAAAATAATGT

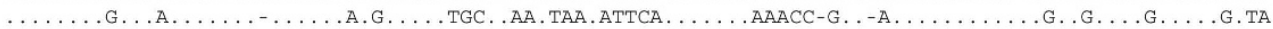
ATTTTAGCTCTAGTGTCCCTAAGAGTTCACACCGTGAGGTCCATGCAGGGCTTTGTGCCGTGCATAGGAGCGGCCTCCCTTCGGATAGTGTTTGCATGGA .

TCAAAATCAAGGTGAATAGGGGAATATATCATAGTAAGTGGGTGAGGGATTTGTGGCAGCACATCCCACGGTCTCTTCCATTAG . . . . . . . . . . ...T.................. .

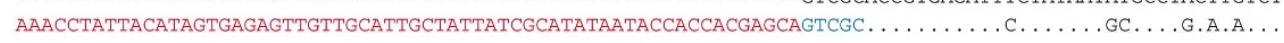

GQ326556 GCCCTGGAGCGAC-GTTCCCTTCGGAGGGTTGTATTATAGGATTCGGAACACCGCGAACTCCAAATCTGGATAGGTTTTCTTAGATTAGTTTTCATAAACW278-Y

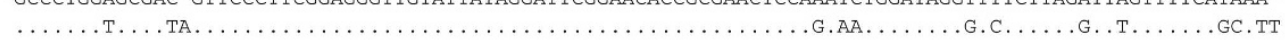

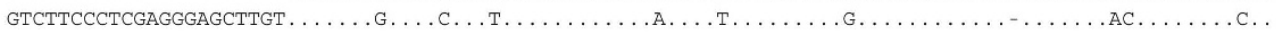

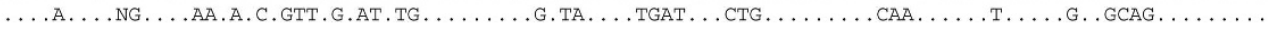

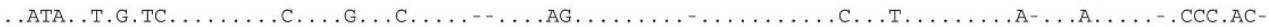

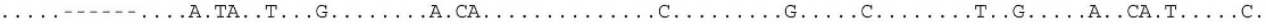
CCGAACTAGTCGGGATGCATATGATCGATGGATAAAAGCTAATGAAAAAGCCCGTGTCTACATTCTTGCCAGCATGTCTGATGTATTGGCAAAGAAACAT

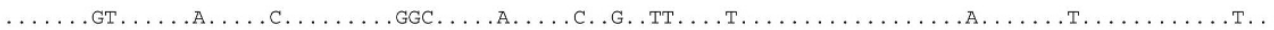

GAATCCTTAGCCACGGCTAAAGAGATTATGGATTCATTAAGGGGAATGTTTGGGCAACCAGAATGGTCCTTAAGACACGAGGCAGTCAAATACATTTACA

GQ326556 CW278-Y

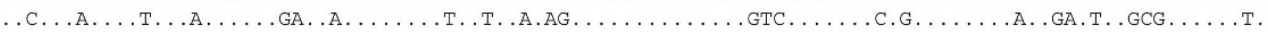

Figure 1 Alignment of CW278-Y with a sequence of a Copia-like retrotransposon from Cucumis. The alignment length is 2340 bp and the length of each sequence is given in brackets at the end. The solo-LTR is in red, the flanking repeats are in blue and the start codon of the retrotransposon is in green (at position 2197 of the alignment). The sequence of the element from Cucumis (GQ326556) presented here is the reverse complement of the sequence in GenBank. 


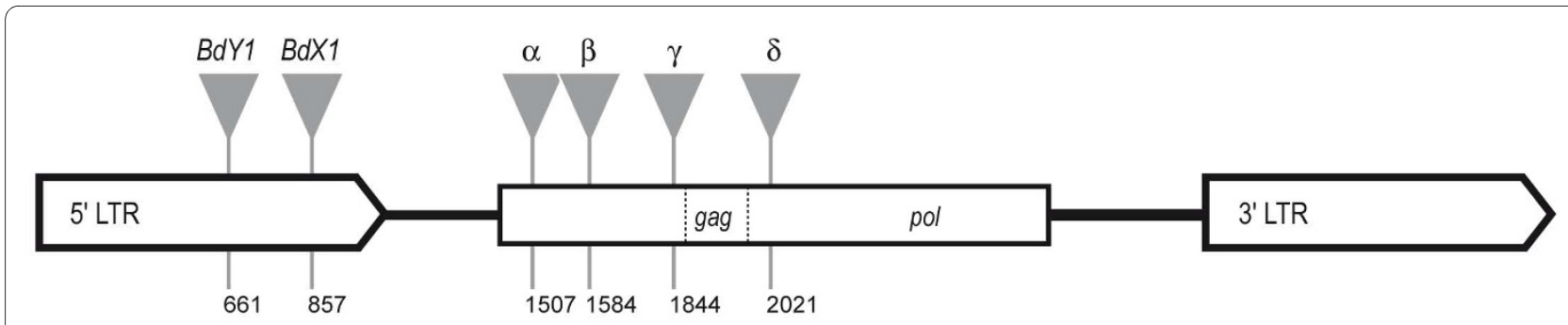

Figure 2 Locations of the solo-LTRs within the RLC-sequence. Schematic representation illustrating the locations of the paralogous solo-LTRs. The position numbers at which each insertion begins are in reference to the consensus sequence of RLC_XXX_Bryonia (RLC-sequence), the common Copia-like element in which the solo-LTRs are found. The coding region begins at bp 1131.

Southern blot analysis on male and female plants using $1110 \mathrm{bp}$ of the $5^{\prime}$ ORF containing the Gag domain as a hybridization probe revealed no significant differences between the two sexes. All digests exhibit strong signals showing the high copy number of these elements in both males and females. Because of EcoRI and HindIII restriction sites in the 5'-LTR and in the coding region, digestion with these enzymes results in single bands of, respectively, $2 \mathrm{~kb}$ and $4.4 \mathrm{~kb}$ (Figure 3a). The distinct fragments observed by HaeIII restriction (Figure 3a) are compatible with the single HaeIII recognition site in the 5'-LTR revealed by sequencing.

A more complex profile was observed in Southern blots that used the solo-LTR as the probe (Figure 3b). Hybridization patterns in all digests revealed strong smears over the whole lane rather than strong bands, suggesting the existence of many copies of the solo-LTR or the corresponding transposable element in random positions in the genome. However, some digests, such as HindIII, show a degree of conservation of this LTR family by the presence of a few, more intense bands of varying sizes. These results are consistent with results from rice using

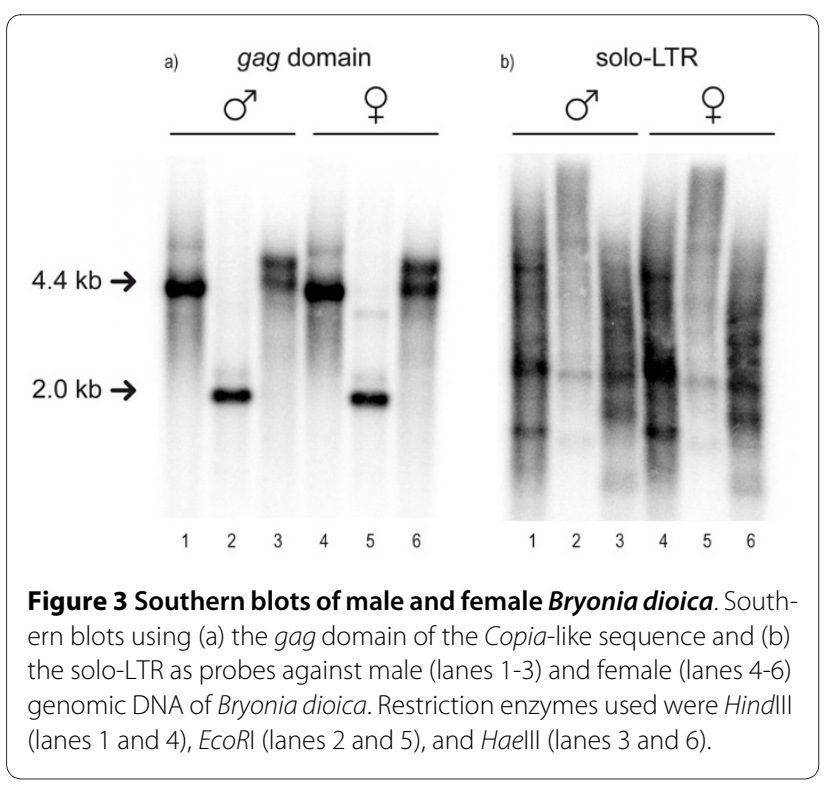

Southern blots, which found some elements to exist in relatively low copy numbers $[35,36]$, and with the observation that LTR retrotransposons are otherwise highly represented in the genome of plants [11].

\section{Discussion}

The first male-linked molecular marker in Bryonia dioica, reported by Oyama et al. [29], is the result of the insertion of one LTR-retrotransposon into another and the subsequent conversion of the inserted element into a solo-LTR. In our earlier paper, a second sequence was also recovered from both males and females that appeared to have an insert relative to the male-linked marker $B d Y 1$ but was otherwise alignable. This second sequence appears to result from a paralogous insertion of the element that gave rise to the solo-LTR about $200 \mathrm{bp}$ downstream of the male-linked one. Since the reverse SCAR primer lies inside the solo-LTR, the PCR primerbinding site is effectively moved $200 \mathrm{bp}$ downstream, giving rise to the second PCR band.

A minimum of four other, presumably paralogous, soloLTRs appear in the underlying retrotransposon sequence. Such multi-layered insertion events have also been observed in maize [14] and probably result from the tendency of LTR-retrotransposons to insert into other LTRretrotransposons [37]. Since solo-LTRs are the remnants of intact LTR-retrotransposons that were removed via unequal homologous recombination, this means that the underlying Copia-like retrotransposon is older than the solo-LTR. Considering that transposable elements are silenced and begin to accumulate mutations quickly [24], one probable reason why we cannot identify the proteinase domain of the Copia-like element is that the necessary motif is too degraded.

Methylation is thought to be important in regulating both the activity of transposable elements and recombination. Most of the transposable elements in plant genomes have been heavily methylated, and this is thought to be the mechanism by which the host genome deactivates the transposons [24]. At the same time, methylation is known to suppress recombination [38], the 
mechanism by which transposable elments would otherwise be eliminated from the genome. With respect to the evolution of sex chromosomes, the suggestion has been made that the accumulation of transposable elements, through their tendency to become methylated, may be what leads to the initial suppression of recombination and allows for the linkage of sex-determining genes [39]. The suppression of recombination would also prevent the removal of transposon sequences, causing the nonrecombining gonosome (e.g., the Y-chromosome) to appear to accumulate transposable element sequences.

Stress is known to be an activator of LTR-retrotransposons [24] and may relate to the repeated shifts between monoecy and dioecy among the ten species of Bryonia [27]. The range expansion of $B$. dioica into northern Europe following the last glacial maximum [28] could have been associated with cold stress and enhanced transposon activity. Population genetic analyses of the male-linked marker $B d Y 1$ throughout northern and southern Europe seemed to detect ongoing recombination in southern Europe [29]. It could be, however, that the recombination detected is actually a signal of transposition, given that the region flanking $B d Y 1$ is a hybrid of the underlying Copia-like element and the inserted solo-LTR.

\section{Conclusions}

The interesting aspect of the solo-LTR indel event described here is that it is male-linked and located in the non-recombining region of the Y-chromosome. That our walk along the Y-chromosome recovered sequence of a Copia-like retrotransposon is congruent with the prevalence of this element in the plant genome, and nesting of LTR-retrotransposons within each other has also been seen previously [14]. However, in light of current theory about sex chromosome evolution, these results may help illuminate the early steps from autosome to sex chromosome; they imply that the recombination event leading to the solo-LTR and the suppression of recombination that would allow this area to be a male-linked marker occurred close together in evolutionary time. Whether the two events are merely correlated or causally linked remains to be clarified. Nevertheless, that a solo-LTR, formed by recombination, is male-linked and characterizes the Y-chromosome, where recombination should be suppressed, provides an interesting insight into the early stages of the evolution of sex chromosomes.

\section{Methods}

\section{Development of male-linked SCAR marker}

The development of the original male-linked SCAR marker $(B d Y 1)$ that served as the anchor point for the chromosome walking was described in Oyama et al. [29] and is only summarized here. An AFLP library was cre- ated for each sex of Bryonia dioica that pooled DNA from eight individuals. This library was filtered and screened for male-linked bands. Putative male-linked bands were confirmed by repeating the procedure for several individuals of each sex. These male-linked bands were isolated and sequenced. SCAR primers were designed from the AFLP sequence, and PCR was performed using these primers and genomic DNA from multiple male and female individuals of $B$. dioica and other Bryonia species.

\section{Sequencing the 5 ' and 3 ' regions flanking $B d Y 1$}

DNA was extracted from young Bryonia dioica male and female leaves following the cetyltrimethylammonium bromide (CTAB) based method [40]. To determine 5'and 3'-flanking sequences of $B d Y 1$, a library was constructed with the GenomeWalker Universal Kit (Clontech Laboratories, Saint-Germain-en-Laye, France) according to the manufacturer's protocol. Briefly, genomic DNA was digested with EcoRV, DraI and SspI and DNA fragments were ligated with a GenomeWalker adaptor supplied in the kit. This GenomeWalker library was used as a template for PCR with an adaptor primer and a gene-specific primer. The PCR mixture was diluted and used as a template for a secondary or "nested" PCR with the nested adaptor primer and a nested gene-specific primer. Cycle conditions for the first PCR: 1 cycle of $3 \mathrm{~min}$ at $94^{\circ} \mathrm{C}, 39$ cycles of $30 \mathrm{~s}$ at $94^{\circ} \mathrm{C}, 1 \mathrm{~min}$ at $59^{\circ} \mathrm{C}$ and $3 \mathrm{~min}$ at $68^{\circ} \mathrm{C}$ for amplification, and 1 cycle of $10 \mathrm{~min}$ at $68{ }^{\circ} \mathrm{C}$ for final extension. Cycle conditions for the second PCR: 5 cycles of $25 \mathrm{~s}$ at $94^{\circ} \mathrm{C}, 3 \mathrm{~min}$ at $72^{\circ} \mathrm{C}, 20$ cycles of $25 \mathrm{~s}$ at $94^{\circ} \mathrm{C}, 3$ min at $67^{\circ} \mathrm{C}$ for amplification and $67^{\circ} \mathrm{C}$ for $10 \mathrm{~min}$ after the final cycle. All PCR mixtures contained 1.25 Units Taq DNA polymerase (QIAGEN GmbH, Hilden, Germany [Qiagen]), $1 \times$ Taq buffer, $15 \mathrm{mM} \mathrm{MgCl}_{2}, 10 \mathrm{mM}$ of each dNTP and 10 pmol of each primer in a total volume of $50 \mu$ l. Gene-specific primers (GSP) are listed in Table 1. Amplicons were purified for sequencing and sequencing reactions were performed with the BigDye Terminator v3.1 cycle sequencing kit (Applied Biosystems, Foster City, California $[\mathrm{ABI}])$. The cycle sequencing products were cleaned by Sephadex G-50 gel filtration (GE Healthcare, Freiburg, Germany) and fragments were separated on an ABI 3130 Genetic Analyser capillary sequencer. Sequences were assembled and edited with Sequencher 4.2 (Gene Codes Cooperation, Ann Arbor, Michigan).

\section{Southern Analysis}

For Southern analysis, total cellular DNA from Bryonia dioica male and female tissues were extracted using the protocol of Fulton et al. [41]. Equal amounts of DNA were digested with HindIII, EcoRI and HaeIII, separated electrophoretically on $1 \%$ agarose gels, and transferred to a Biodyne B nylon membrane (Pall Gelman Laboratory, Ann Arbor, Michigan). Hybridizations were performed under high stringency conditions for $24 \mathrm{~h}$ at $60^{\circ} \mathrm{C}$ using 


\section{Table 1: Primer sequences used for chromosome and genome walking}

\begin{tabular}{ll}
\hline $\begin{array}{l}\text { Primer sequences for chromosome walking on the } \text { Y- } \\
\text { chromosome }\end{array}$ \\
\hline GSP7 & 5'-TGCATTCTACAAGCTATCACCGACC \\
GSP8 & 5'-AGAGACCGTGGGACATGGTGTCACATACCTC \\
GSP16 & 5'-GCCGTGCATAGGAGCAGACTC \\
GSP17 & 5'-AGTGGGTGAGAGGTATGTGACACCATGTC \\
GSP18 & 5'-ACGGTCTCTTCCATTAGGTCGGTGATAGC \\
GSP20 & 5'-AAGGAAGAATTGCGCCAGCAC \\
GSP21 & 5'-CAGCTGATCAAGAGTTACTCCCAGTCAACTG \\
GSP22 & 5'-TCCGGATGCGGTGGCGCATTG \\
GSP23 & 5'-AATGCGCCACCGCATCCGGAC \\
GSP24 & 5'-TCAGGGACCATGACGGTTC \\
& \\
\hline Primer sequences for genome walking to recover \\
retrotransposon sequence \\
\hline GSP15 & 5'-GGTCTCTTCCATTAGGTCGCACCGTGAG \\
GSP17 & 5'-AGTGGGTGAGAGGTATGTGACACCATGTC \\
GSP19 & 5'-ACTACCCTTCGTAGATGTTGC \\
GSP19-1 & 5'-TGCAATCAAGACGGGCATTGG \\
GSP19-7 & 5'-CCTTAGAGCTCGTACATTCGGAC \\
GSP19-8 & 5'-CCCAGCAGAACGGTGTATCAG \\
GSP19-9 & 5'-TGGATATGGTTCGCTCTATGATGAGC \\
GSP19-12 & 5'-ATGGTTCGCTCTATGATGAGC \\
GSP19-14 & 5'-TGATGTGGATCGTGACCAGTGG \\
\hline & \\
\hline
\end{tabular}

$[\alpha-32 \mathrm{P}] \mathrm{dCTP}$-radiolabeled probes. Probes were obtained through PCR amplification using primer pair 5'-GTGCAACTCTTAGCTTCCGAG/5'-TTCGGCTGAGACAACCTCTCC for the gag domain and 5'-ACGGTCT CTTCCATTAGGTCGGTGATAGC/5'-AAGAGACCCTATCCATTTCTGGAGTTCG for the solo-LTR. PCRs were carried out under the same conditions as used in the first PCR for genome walking. The products were purified with QIAquick gel extraction kit (Qiagen).

Competing interests

The authors declare that they have no competing interests.

\section{Authors' contributions}

RKO helped conceive and plan the study, analyzed the data, and drafted the manuscript. MVS gathered the sequence and Southern blot data, analyzed the data, and helped draft the manuscript. SSR conceived of the project and also worked on the manuscript. All authors have read and approved the final manuscript.

\section{Acknowledgements}

This work was supported by grant RE603/4-2 of the German Research Foundation (DFG)

\section{Author Details}

Systematic Botany \& Mycology, Ludwig-Maximilians-Universität (LMU Munich), 80638 Munich, Germany

Received: 19 May 2010 Accepted: 14 June 2010

Published: 14 June 2010

\section{References}

1. Renner SS, Ricklefs RE: Dioecy and its correlates in the flowering plants. American Journal of Botany 1995, 82(5):596-606.

2. Jamilena M, Mariotti B, Manzano S: Plant sex chromosomes: molecular structure and function. Cytogenetic and Genome Research 2008, 120(34):255-264.

3. Vyskot B, Hobza R: Gender in plants: sex chromosomes are emerging from the fog. Trends in Genetics 2004, 20(9):432-438.

4. Charlesworth D, Charlesworth B, Marais G: Steps in the evolution of heteromorphic sex chromosomes. Heredity 2005, 95(2):118-128.

5. Bergero R, Forrest A, Kamau E, Charlesworth D: Evolutionary strata on the $\times$ chromosomes of the dioecious plant Silene latifolia: Evidence from new sex-linked genes. Genetics 2007, 175(4):1945-1954.

6. Ming R, Wang JP, Moore PH, Paterson AH: Sex chromosomes in flowering plants. American Journal of Botany 2007, 94(2):141-150.

7. Charlesworth B, Charlesworth D: Model for evolution of dioecy and gynodioecy. American Naturalist 1978, 112(988):975-997.

8. Spigler RB, Lewers KS, Main DS, Ashman TL: Genetic mapping of sex determination in a wild strawberry, Fragaria virginiana, reveals earliest form of sex chromosome. Heredity 2008, 101(6):507-517.

9. Armstrong SJ, Filatov DA: A cytogenetic view of sex chromosome evolution in plants. Cytogenetic and Genome Research 2008, 120(34):241-246.

10. Kejnovsky E, Hobza R, Cermak T, Kubat Z, Vyskot B: The role of repetitive DNA in structure and evolution of sex chromosomes in plants. Heredity 2009, 102(6):533-541.

11. Kejnovsky E, Leitch IJ, Leitch AR: Contrasting evolutionary dynamics between angiosperm and mammalian genomes. Trends in Ecology \& Evolution 2009, 24(10):572-582.

12. Bennetzen $\mathrm{J}$ : The structure and evolution of angiosperm nuclear genomes. Current Opinion in Plant Biology 1998, 1(2):103-108.

13. Kuykendall D, Shao J, Trimmer K: A nest of LTR retrotransposons adjacent the disease resistance-priming gene NPR1 in Beta vulgaris L. U.S. Hybrid H20. Int J Plant Genomics 2009, 2009:576742.

14. SanMiguel P, Gaut BS, Tikhonov A, Nakajima Y, Bennetzen JL: The paleontology of intergene retrotransposons of maize. Nature Genetics 1998, 20(1):43-45.

15. Devos KM, Brown JKM, Bennetzen JL: Genome size reduction through illegitimate recombination counteracts genome expansion in Arabidopsis. Genome Research 2002, 12(7):1075-1079.

16. Vitte C, Panaud O: Formation of solo-LTRs through unequal homologous recombination counterbalances amplifications of LTR retrotransposons in rice Oryza sativa L. Molecular Biology and Evolution 2003, 20(4):528-540

17. Bergero R, Forrest $A$, Charlesworth $D$ : Active miniature transposons from a plant genome and its nonrecombining $Y$ chromosome. Genetics 2008, 178(2):1085-1092

18. Hobza R, Lengerova M, Svoboda J, Kubekova H, Kejnovsky E, Vyskot B: An accumulation of tandem DNA repeats on the $Y$ chromosome in Silene latifolia during early stages of sex chromosome evolution. Chromosoma 2006, 115(5):376-382.

19. Matsunaga S, Yagisawa F, Yamamoto M, Uchida W, Nakao S, Kawano S: LTR retrotransposons in the dioecious plant Silene latifolia. Genome 2002, 45(4):745-751

20. Obara M, Matsunaga S, Nakao S, Kawano S: A plant Y chromosome-STS marker encoding a degenerate retrotransposon. Genes \& Genetic Systems 2002, 77(6):393-398.

21. Mariotti B, Manzano S, Kejnovsky E, Vyskot B, Jamilena M: Accumulation of Y-specific satellite DNAs during the evolution of Rumex acetosa sex chromosomes. Molecular Genetics and Genomics 2009, 281(3):249-259.

22. Navajas-Perez R, Schwarzacher $T$, de la Herran $R$, Rejon CR, Rejon MR, Garrido-Ramos MA: The origin and evolution of the variability in a $Y$ specific satellite-DNA of Rumex acetosa and its relatives. Gene 2006, 368:61-71. 
23. Sakamoto K, Abe T, Matsuyama T, Yoshida S, Ohmido N, Fukui K, Satoh S: RAPID markers encoding retrotransposable elements are linked to the male sex in Cannabis sativa L. Genome 2005, 48(5):931-936.

24. Liu B, Wendel JF: Retrotransposon activation followed by rapid repression in introgressed rice plants. Genome 2000, 43(5):874-880.

25. Correns C: Über die dominierenden Merkmale der Bastarde. Berichte der Deutschen Botanischen Gesellschaft 1903, 21:133-147.

26. Correns C: Die Bestimmung und Vererbung des Geschlechtes, nach Versuchen mit höheren Pflanzen. Verhandlungen der Gesellschaft deutscher Naturforscher und Ärzte 1907, 1907:794-802.

27. Volz SM, Renner SS: Hybridization, polyploidy and evolutionary transitions between monoecy and dioecy in Bryonia (Cucurbitaceae). American Journal of Botany 2008, 95(10):1297-1306.

28. Volz SM, Renner SS: Phylogeography of the ancient Eurasian medicinal plant genus Bryonia (Cucurbitaceae) inferred from nuclear and chloroplast sequences. Taxon 2009, 58(2):550-560.

29. Oyama RK, Volz SM, Renner SS: A sex-linked SCAR marker in Bryonia dioica (Cucurbitaceae), a dioecious species with XY sex-determination and homomorphic sex chromosomes. Journal of Evolutionary Biology 2009, 22(1):214-224.

30. Altschul SF, Gish W, Miller W, Myers EW, Lipman DJ: Basic Local Alignment Search Tool. Journal of Molecular Biology 1990, 215(3):403-410

31. Zhang Z, Schwartz S, Wagner L, Miller W: A greedy algorithm for aligning DNA sequences. Journal of Computational Biology 2000, 7(1-2):203-214.

32. Wicker T, Matthews DE, Keller B: TREP: a database for Triticeae repetitive elements. Trends in Plant Science 2002, 7(12):561-562.

33. Wicker T, Sabot F, Hua-Van A, Bennetzen JL, Capy P, Chalhoub B, Flavell A, Leroy $P$, Morgante $M$, Panaud $O$, et al:: A unified classification system for eukaryotic transposable elements. Nature Reviews Genetics 2007 8(12):973-982.

34. Marchler-Bauer A, Bryant SH: CD-Search: protein domain annotations on the fly. Nucleic Acids Research 2004, 32:W327-W331.

35. Wang SP, Liu N, Peng KM, Zhang QF: The distribution and copy number of Copia-like retrotransposons in rice (Oryza sativa L.) and their implications in the organization and evolution of the rice genome. Proceedings of the National Academy of Sciences of the United States of America 1999, 96(12):6824-6828.

36. Wang SP, Zhang QF, Maughan PJ, Maroof MAS: Copia-like retrotransposons in rice: Sequence heterogeneity, species distribution and chromosomal locations. Plant Molecular Biology 1997, 33(6):1051-1058.

37. Bennetzen JL: Transposable element contributions to plant gene and genome evolution. Plant Molecular Biology 2000, 42(1):251-269.

38. Gorelick R: Evolution of dioecy and sex chromosomes via methylation driving Muller's ratchet. Biological Journal of the Linnean Society 2003 80(2):353-368

39. Gorelick R: Theory for why dioecious plants have equal length sex chromosomes. American Journal of Botany 2005, 92(6):979-984.

40. Doyle JJ, Doyle JL: A rapid DNA isolation procedure for small quantities of fresh leaf material. Phytochemical Bulletin 1987, 19:11-15.

41. Fulton TM, Chunwongse J, Tanksley SD: Microprep protocol for extraction of DNA from tomato and other herbaceous plants. Plant Molecular Biology Reporter 1995, 13(3):207-209.

\section{Submit your next manuscript to BioMed Central} and take full advantage of:

- Convenient online submission

- Thorough peer review

- No space constraints or color figure charges

- Immediate publication on acceptance

- Inclusion in PubMed, CAS, Scopus and Google Scholar

- Research which is freely available for redistribution

Submit your manuscript at www.biomedcentral.com/submit
C Biomed Central 